\title{
Malva sylvestris/Alcea digitata Herbal Compound
}

National Cancer Institute

\section{Source}

National Cancer Institute. Malva sy/vestris/Alcea digitata Herbal Compound. NCI

Thesaurus. Code C131822.

A traditional Persian medicine composed of Malva sylvestris (M. sylvestris) and Alcea digitata (A. digitata; Boiss), with potential use for the treatment of radiation- and chemotherapy-induced xerostomia, and potential immunomodulating, anti-oxidant and antimicrobial activities. Upon administration to the mouth, M. sylvestris/A. digitata herbal compound may lubricate the mouth and throat, and may improve the symptoms of dry mouth, thereby preventing tooth decay and improving chewing, eating, tasting, swallowing, and speaking. M. sylvestris/A. digitata herbal compound may also improve mucositis by modulating the immune system, thereby preventing infection, reducing inflammation and promoting wound healing. 